\title{
Semantic Relation Modeling and Representation for Problem-Solving Ontology-Based Linguistic Resources: Issues and Proposals
}

\author{
Francisco Alvarez ${ }^{1}$, Antonio Vaquero ${ }^{2}$, Fernando Sáenz ${ }^{2}$, Manuel de Buenaga ${ }^{3}$ \\ ${ }^{1}$ Universidad Autónoma de Sinaloa, Ángel Flores y Vicente Riva Palacios, s/n, \\ C.P. 80000 , Culiacán, Sinaloa, México \\ ${ }^{2}$ Universidad Complutense de Madrid, Facultad de Informática, Departamento de \\ Ingeniería del Software e Inteligencia Artificial, C/ Prof. José García Santesmases, \\ s/n, E-28040, Madrid, Spain \\ ${ }^{3}$ Universidad Europea de Madrid, Departamento de Sistemas Informáticos, 28670 \\ Villaviciosa de Odón. Madrid, Spain \\ fjalvare@fdi.ucm.es, \{vaquero, fernan\}@sip.ucm.es, buenaga@uem.es
}

\begin{abstract}
Semantic relations are an important element in the construction of ontologies and models of problem domains. Nevertheless, they remain underspecified. This is a pervasive problem in Software Engineering and Artificial Intelligence. Consequently, we find semantic links that can have multiple interpretations in ontologies that support information systems, semantic data models with abstractions that are not enough to capture the relation richness of problem domains, and improperly structured taxonomies. However, if provided with precise semantics, some of these problems can be avoided, and meaningful operations can be performed on them. In this paper we present some insightful issues about the modeling and representation of semantic relations that include the available taxonomy structuring methodologies as well as the initiatives aiming to provide relations with precise semantics. Furthermore, we explain and propose the notion of control and verification of relations as a means to properly structure ontologies.
\end{abstract}

\section{Introduction}

It has been suggested that ontologies can provide benefits for almost any knowledge based information system. Among these suggested benefits is the ability to reuse an ontology developed for one task in another task. However, there is one neglected aspect that severely compromises the reusability and integration of ontologies: semantic relations. Although they hold together the entities that represent a domain and shape its structure, semantic relations have not been given the attention they deserve. The focus has been on concepts, their properties and the operations that can be performed on them, rather than on the semantics of relations.

Hence, in order to avoid some of the problems that arise from this neglectful attitude, semantic relations (whether taxonomic or not) must be provided with finegrained semantics. This is an important issue, in particular for the development of problem-solving ontology-based information systems. Tackling a precise task inside a 
given domain requires precise semantics, not only at the concept level but also at the relation level, that is, we should be able to describe relations in the same breadth of detail as concepts.

As part of the SINAMED and ISIS projects [1], we have pointed out in [2, 3] the need to: a) provide the relations used in ontologies with definite semantics and b) to develop ontology-based linguistic resources (OBLR) following a software engineering approach. Nonetheless, although a set of ideas has been proposed for the representation of relations and their semantics, the results of our research stem mainly from an analysis of "task-neutral" linguistic resources (LR) presented in [4].

In this paper, we present an analysis of the state-of-the-art in the representation of semantic relations in Software Engineering (SE) and Artificial Intelligence (AI), including the available methodologies to structure taxonomies. Our goal is to gain a better insight of this normally neglected topic, underline its importance for the development of information systems, and determine if the available technology is well-suited to provide semantic relations with the level of granularity that problemsolving ontology-based information systems need. Furthermore, we aim at covering some of the blanks left in the current research regarding the representation of relations and their semantics, and its possible application to the construction of domain and task dependent ontologies and OBLR.

The rest of the paper is organized as follows. In section 2, the limitations of SE techniques in the representation of relations are described. In section 3, the overstress of concepts in detriment of relation representation in AI and taxonomy structuring methodologies is shown. In section 4, the current initiatives to provide relations with explicit semantics are presented. In Section 5, control and verification is introduced as a meaningful operation based on the intrinsic semantics of relations. Finally, in section 6 , some conclusions and future work are outlined.

\section{Semantic Relations in Software Engineering}

Semantic relations (relationships in the SE vocabulary) are a key component of vital design artefacts such as Entity-Relationship (E-R) models and object-class diagrams (OCD). However, they only capture a limited set of relationships, leaving much of the domain's relationship structure out of the design [5].

In the E-R model [6] the model of the problem is represented by identifying its entities, properties and relationships. In this model, relationships are classified among entities as binary, n-ary, or recursive. Nonetheless, although they are depicted on the E-R diagram, the amount of information they convey is rather limited, that is, the model itself only provides minimal information describing the relationships (i.e. mainly the cardinality among entities).

In OCD [7, 8], objects are organized by their similarities into classes that describe a set of objects having the same attributes and behavior patterns. In these models, relationships among classes can be classified under three basic categories denoting: a) generalization-specialization; b) whole-part/aggregation and c) an association among otherwise unrelated classes.

In OCD, generalization and aggregation types of relationships are strongly defined among classes. Nevertheless, all other types of relationships that exist in the problem 
domain are lumped into the association category and depicted by a name connecting the classes. These names only indicate that a dependency exists but do not explicitly indicate how. Thus, association relationships are identified more implicitly than explicitly. Furthermore, the distinction between aggregation and association is often a matter of state rather than a difference on semantics [9].

In the UML [10] relationships suffer from the same under-specification. The UML categorizes relationships under five categories: association, aggregation (denoting a part-of relationship), dependency, generalization-specialization (denoting an is-a relationship) and realization. As with OCD relationship classification, generalization and aggregation relationships are strongly defined among classes. However, association, dependency and realize relationships have to be identified by looking at the sequence and collaboration diagrams. Furthermore, only a label is used to indicate that these relationships exist. Thus, as in OCD, they are identified more implicitly than explicitly.

Although we have described just a few semantic data models here, several others suffer from the same problem: namely minimal information describing relationships. As can be seen, relations are neglected in favor of object or entity representation. However, as it will be shown next, this is not inherent to SE; it is also a pervasive phenomenon in AI.

\section{Semantic Relations in Artificial Intelligence}

In AI, we find that knowledge representation formalisms (e.g. DAML and OWL) are intended to describe the terminology of a domain in terms of classes/concepts describing sets of individuals and properties/roles relating these [11]. However, although in the above formalisms is possible to make statements about a set of concepts, such as to declaratively specify that two classes are disjoint; analogous declarative statements are not possible for relations. This also comprises the assignment of properties, while concepts are assigned with as many properties as needed, the same level of precision cannot be applied to semantic relations.

Furthermore, the modeling effort is done through the construction of subsumption hierarchies among the defined classes and properties, that is, taxonomic reasoning is restricted to a generalization-based one. This is partially caused by the lack of conclusive mechanisms to reason along other types of relations [12], and because, generalization was initially regarded as the primary mechanism for mastering the complexity of domains.

Consequently, there is a subsumption overload that has led to the misuse, confusion and conflation of semantic relations due to the lack of analysis to: a) distinguish between the different relations to be used in the representation of a domain and b) precise the semantics of these relations. For instance, without such an analysis what we have are ontological resources that have very general or under-specified relations that cannot be adequately interpreted [13], resources whose relations are subject to multiple interpretations [11], resources where the semantics of the relations are not fine-grained enough as to allow to differentiate between two relations that are close in meaning but are not the same (e.g. the is-a and hypernym relations [14]), and improperly structured taxonomies. 
For instance, in SNOMED-RT (a clinical terminology developed by the College of American Pathologists), the concept "testis" subsumes (correctly) the concepts "left testis", "right testis" and "undescended testis", but also "both testes". We could accept "both testes" as being part-of another concept (e.g. "Testes") denoting the mereological sum of the left and right testes, but hardly as being a "Testis". In this case, the mistake is introduced manually by one of the ontology developers who incorrectly assigned a direct is-a link. Figure 1 shows this mistake.

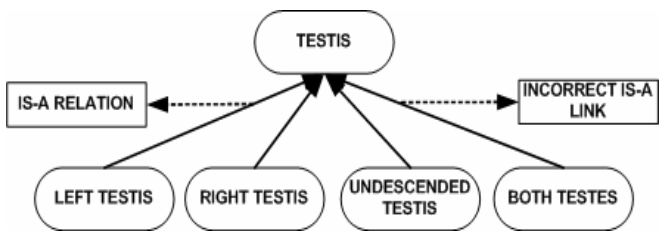

Fig. 1. Wrong Modeling Choice

Similarly and again in SNOMED-RT, "Hair" subsumes (correctly) "Vellus Hair" and "Terminal Hair", but also "Hair Shaft". However, a quick look in a dictionary will tell us that "Hair Shaft" is not a kind of "Hair" but part-of it.

This kind of problems has received most of the attention in AI. However, as it will be seen, the available approaches for taxonomy structuring focus more on concepts and their properties rather than on relations and their semantics. Moreover, although the ontology structuring problems represent a serious obstacle in the development and integration of ontological resources, there are only two proposals that actually deal with it: The OntoClean [15] and [16] methodologies. These methodologies are similar but at the same time, as it will be seen below, follow opposite approaches to attain the same goal.

\subsection{The Metaphysical Approach of OntoClean}

OntoClean is grounded on the philosophical and metaphysical ideas of essentialism [17] that state that for any specific kind of entity (e.g. a tiger), it is theoretically possible to specify a finite list of properties (e.g. the rigidity, identity and unity metaproperties of OntoClean) all of which any entity must have to belong to a specific group or natural kind (e.g. see the table of properties and the taxonomy of kinds in [15].

It is also influenced by the ideas of psychological essentialism [18] that enunciate that the world is divided into essences from which preset associated properties can be inferred (e.g. the metaproperties mentioned above), and that these properties play a key role in our everyday reasoning and categorization tasks by backing-up our inferences about kind membership.

Hence, OntoClean can be understood as a reasoning heuristic and inference system that establishes that the compatibility between the metaproperties defining the essence of concepts determine if a concept can subsume another and vice versa.

Nevertheless, a global theory of reference and categorization, independent of any domain and task, like the one OntoClean provides is not possible. Recent work in 
cultural psychology has shown systematic cognitive differences between East Asians and Westerners, and some work indicates that this extends to intuitions about philosophical cases [19].

Furthermore, it is interesting to note that just like in OCD and the UML, the generalization (is-a) and aggregation (part-of) relations are strongly defined in OntoClean. This is done through an axiomatization (see the set of definitions in [15]) that presents itself as an absolute truth. However, just like OCD, any other relation that falls out of the scope of the methodology cannot be described nor controlled. Hence, the limited set of abstractions wherewith OntoClean pretends to master the complexity of any domain and structure an ontology is not enough. Figure 2 shows the OntoClean approach.

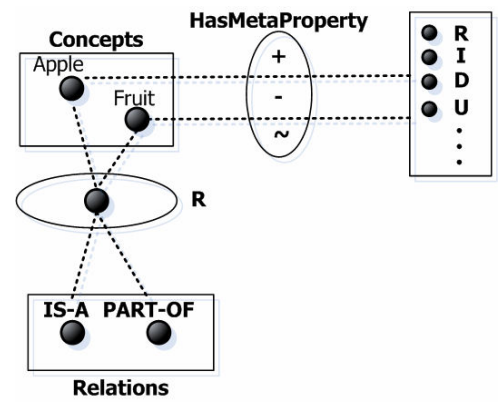

Fig. 2. The OntoClean Approach

\subsection{The Linguistic Approach of Archonte}

Archonte relies on the work of [20] that states that even for well-defined domains, the norms that fix the meaning of a word and of its reference (e.g. its concept) cannot be foreseen, and that the meaning of words is immanent to a given situation and context of usage. Archonte claims to provide concepts with a domain and task-dependent meaning by means of the similarities and differences that a linguistic unit has with other neighboring units in the same context of usage, and that this differences and similarities can be found by analyzing a corpus

This method requires the use of terminological extraction tools to discover candidate concepts from the corpus. Once this is done, it uses a set of principles [16] to create and structure a backbone taxonomy where the differences and similarities between concepts are expressed in natural language. This is much in the spirit of the taxonomies found in machine-readable dictionaries. Nonetheless, while these taxonomies are twisted, Archonte provides a manual method to create properly structured linguistic taxonomies from words with the aid of an expert.

The principles are the following: a) similarity with parent (SWP); b) similarity with siblings (SWS); c) difference with siblings (DWS) and d) difference with parent (DWP). Since these principles are attached to concepts, herein lies the similarity with OntoClean. In order to structure an ontology, concepts (not relations) must have a set of (meta) properties that determine if a semantic link can exist between any two concepts. Nonetheless, although Archonte claims to be domain and task-dependent, it 
is clear that it is only domain-dependent. Concepts and relations are obtained by processing a domain-dependent corpus, but the corpus itself is independent of any task, as well as the set of properties used to arrange the concepts. Figure 3 illustrates the Archonte approach.

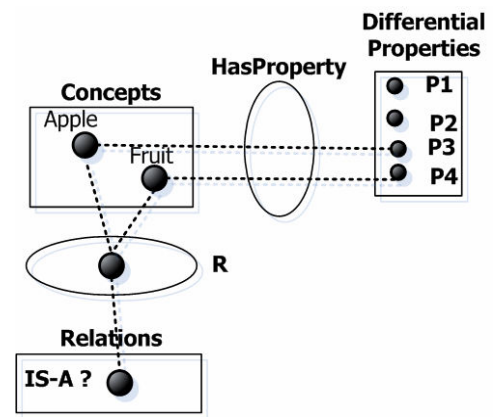

Fig. 3. The Archonte Approach

Given the evidence, we claim that structuring an ontology cannot be done relying on the properties of concepts. The aforementioned task must be done by putting the entire semantic load on the relations. In the next section, we will try to clarify this last point.

\section{Refining and Describing Semantic Relations}

In [4] we do an analysis of "task-neutral" LR and point out the need to provide relations with intrinsic semantics in order to prevent the taxonomic flaws of these resources, and in $[2,3]$ we propose to divide the semantics of relations into algebraic and intrinsic properties and to apply the principles of SE to the development of OBLR. Nonetheless, several things were left out.

First, do SE and AI provide the tools to supply semantic relations with the level of granularity that is needed for the development of software engineered, domain and task-dependent OBLR? Second, do taxonomy structuring methodologies actually deal with the contents of the semantic link around which the backbone taxonomy is constructed? Third, although the algebraic properties of relations can be well understood in our proposal [2], the intrinsic properties are left unspecified. Hence, what could these intrinsic properties be? What is the meaning they could convey? What would they be useful for?

Sections 2 and 3 provide an answer for the first two questions. As for the third one, relation element theory (RET) provides an answer. As explained in [21], RET is an effort to provide an exhaustive as possible classification (under the form of a taxonomy) of binary semantic relations (here, the reader must notice the resemblance between RET and the relationship classification of OCD), on the basis of the nature of the relation between a parent or domain concept and a child or range concept. However, although it would be very difficult to derive an exhaustive and universally agreed upon taxonomy of relations, a set of relation elements or relation primitives are proposed that could be used to describe and refine the semantics of a relation between two entities. These elements are the following: Composable, Connected, 
Functional, Homeomerous, Intangible, Intrinsic, Near, Separable, Structural and Temporal.

A possible application of these primitives would allow countering the polysemy and synonymy of relations within a same knowledge domain or context. For instance, a relation part-of relating an entity Engine to another entity Car could be defined synonymously in another ontology as "physicalParts", but a machine without prior definitions cannot infer that these two relations are identical. Moreover, providing that we have a suitable algebra, there is the possibility of doing plausible inference [21] by using the semantic primitives of relations to infer new relation instances between sets of entities.

However, although the use of the aforementioned set of primitives can clarify the underlying semantics of relations, and meaningful operations can be performed with them, it seems that these primitives conflate several properties that should be separately and explicitly represented as part of the semantics of a single relation or they simply ignore these properties despite the fact that, as it will be seen below, these properties stem from the definition of the primitive itself.

\subsection{Algebraic and Intrinsic Properties of Relations}

First, the primitive Connected indicates that the domain element is temporally or physically connected to the range element either directly or transitively. Here, direction and transitivity are conflated into one single primitive and made inherent to the primitive itself. This is a mistake, because for a given domain, a relation can be directed and transitive or directed and non-transitive, as demonstrated by [22] in their analysis of semantic relations in the medical domain.

For instance, Perforation-of-Appendix implies a perforation of the Intestine because Appendix is part-of Intestine, but Appendicitis (Inflammation of the Appendix) does not imply an inflammation of the Intestine. Similarly, Glomerulus is part-of Kidney, and Appendix is part-of Intestine. However, although Glomerulonephritis (an inflammation of the Glomerulus) is-a Nephritis (an inflammation of the kidney), an Appendicitis is not an Enteritis (inflammation of the Intestine).

Second, the primitive Intangible denotes that the relation that links the domain and range elements is hierarchical with regard to ownership or mental inclusion. However, it is well-known that hierarchical relations (e.g. is-a and part-of), can have a set of algebraic properties (e.g. asymmetry, irreflexivity, transitivity, etc.) that are useful to make valid syllogistic inferences. Nonetheless, it is unknown (as it is not explicitly stated) if the primitive comprises any of these properties or others.

Third, the Structural primitive specifies that the domain and range elements have a hierarchical relationship in which the domain element is below the range element in the hierarchy. Basically, what this primitive is telling us is that the range subsumes the domain or vice versa. Nevertheless, as with the Intangible primitive the set of algebraic properties related to hierarchical relations are simply ignored.

In spite of this, [21] argues that the scope of these primitives could be restricted to a knowledge domain of interest or to a context within a knowledge domain, in order to avoid the complications that arise when aiming for a universal set of primitives that could describe every relation in every domain. RET, just like OCD, UML, OntoClean 
and Archonte, seeks universality by using a small set of abstractions to model a domain.

In [2], we propose to represent semantic relations in terms of intrinsic and algebraic properties. We achieve several objectives by partitioning the semantics of relations this way: a) to separate those properties needed to make syllogistic inferences (e.g. transitivity) from any domain or task dependent properties (as it will be seen below); b) to avoid making any property or primitive a general definitional property of a relation; c) to allow making fine-grained distinctions for each relation independently of any knowledge representation language.

Nevertheless, it has to be clearly stated that, as opposed to RET, we do not aim for an exhaustive classification of relations nor do we propose a universal set of properties through which any relation can be represented. We just propose to represent relations with well-defined semantics up to the granularity that is needed for the task at hand.

Therefore, for a given domain and task, each relation must be provided with a set of properties defining its semantics. Thus, we have algebraic properties that are domain-independent and intrinsic properties that are domain-dependent and taskdependent. This is necessary for modeling non-formal domains like medicine where we not only need to define relations with a simple tag, but also to qualify or precise the relations that link a part and a whole, a child and a parent, etc. with additional attributes. Some examples taken from the medical domain might help to clarify our point.

\subsubsection{Modeling Relations in the Medical Domain}

In the medical domain, the part-of relation not only states that A is part of B, it also needs a set of properties to precise its meaning within a given context. For example, parts of an organ can be shared (they belong to several anatomical entities) or unshared (they belong to a single anatomical entity). Blood vessels and nerves that branch within a muscle must be considered a part-of that muscle and of the vascular or neural trees to which they belong. In contrast, the fleshy part-of the muscle (made of muscle tissue) and the tendon (made of connective tissue) are unshared.

Similarly, the "adjacent to" relation not only expresses that A is adjacent to B, it also must have a set of attributes defining the adjacency: left, right, inferior, posterior, etc. In figure 4, we can see an example of this. The brachialis is "adjacent to" the bicep tendon but is located behind (posterior to) it. The same applies to any other relation that is necessary to model a domain.

Furthermore, we must "artificially" subdivide a domain according to the demands of a given task. Hence, if for a given anatomical structure, we want to record the site of an injury with precision we must further subdivide it in terms of concepts and relations, in order to capture the granularity of this task.

For instance, as can be seen in figure 4, the biceps muscle has several anatomical parts that are structurally distinct from one another (i.e. a long head, a short head, a belly, a tendon). Nonetheless, if we want to record the site of an injury with precision in this structure, we must distinguish the intracapsular part of the tendon of the long head of the biceps (which is enclosed within the capsule of the shoulder joint) from the extracapsular part of the same tendon. 


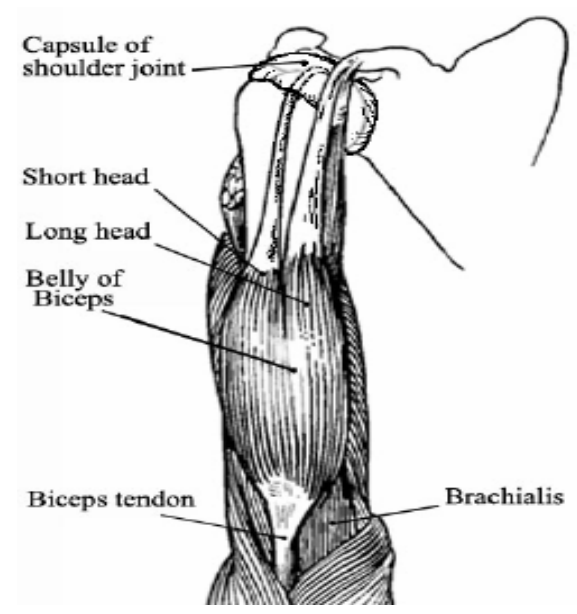

Fig. 4. The Biceps and its Parts

This is precisely what OntoClean, Archonte, RET and even description logics cannot provide: the fine-grained distinctions that a problem-solving task in a given domain requires in terms of relation representation, because the nature and number of relations covering any domain and task is impossible to foresee. Figure 5 shows the proposed approach for relation representation.

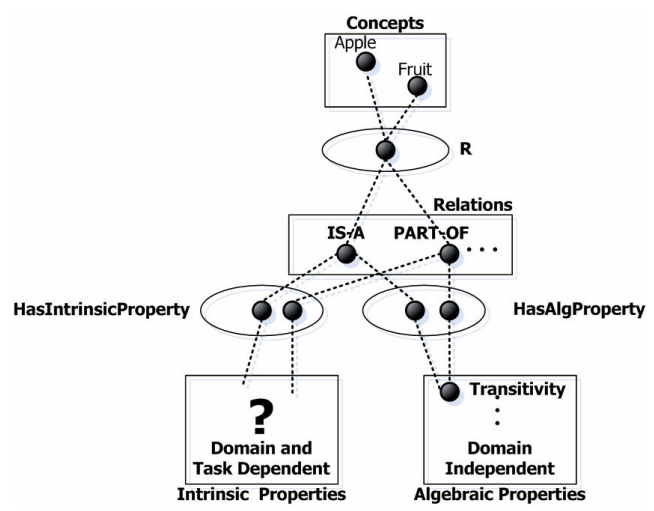

Fig. 5. Algebraic and Intrinsic Properties of Relations

Based on these ideas we introduce the notion of control and verification of semantic relations, a notion that we will try to clarify in the following section.

\section{Control and Verification of Semantic Relations}

Of the many difficulties in building a useful knowledge-based system (KBS), verification is one of the greatest challenges, and as we automate even more and more tasks the need for verification becomes even more crucial [23]. 
As far as semantic relations are concerned, control and verification entails that for a given domain and task, a set of conditions must be established to test whether two concepts can be linked by a given relation. Nevertheless, this can only be enforced but through the use of relations with well-defined semantics highly dependent on the domain and task.

The goal is to properly structure an ontology by using a set of properties that can act as domain constraints, without resorting to the metaphysical universality or to the text-dependent generality of the available taxonomy structuring methods, as they do not take into account the semantics of relations nor the task for which an ontology is being built. Consequently, a complete set of relations must be identified and documented early at the development process by doing an analysis of the domain and the task to be carried out. Moreover, for each relation, its set of intrinsic and algebraic properties must be established as well.

Let us suppose that we are trying to develop an ontology to support a legal information system to "assess claims for immigration" in "Schengen signatory countries". Our domain analysis might show that only the is-a relation is needed to model the problem domain with the following properties: a) asymmetry, reflexivity and transitivity as algebraic properties; b) has borders, has constitution and has central government as domain-dependent intrinsic properties; and c) signed treaty of adhesion and signed Schengen agreement as task-dependent intrinsic properties. Based on these properties, the system could ask meaningful questions to the ontology developer in order to prevent inappropriate modeling choices. Figure 6 illustrates this.

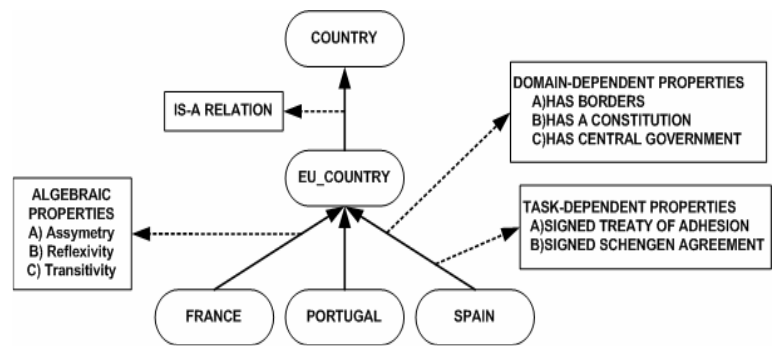

Fig. 6. A Domain Modeled with a Single Relation

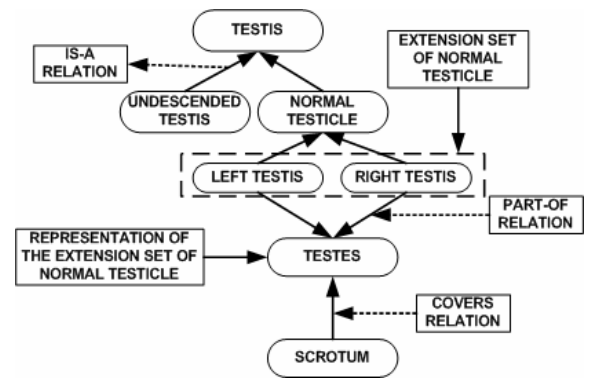

Fig. 7. A Plausible Modeling Choice

With this approach, wrong modeling choices like the one in figure 1 can be avoided and plausible representations like the one in figure 7 (for the testis example in 
section 3) can be obtained. Nevertheless, the concepts and relations shown in this figure, as discussed in the previous section, would represent facts about the domain, and some would be needed to support a given task.

\section{Conclusions and Future Work}

Semantic relations are an important part in the construction of the model of a problem domain in SE and AI. However, they have been neglected in favor of concepts or entities.

In SE, semantic data models have an emphasis on entity representation. Relationship representation is done through a. small set of fundamental abstractions and when a relationship falls outside their scope it is loosely defined and represented by a tag, or lumped under a category that does not account for its semantics.

In AI, the level of semantic precision in terms of properties and attributes, as well as the operations that can be performed on semantic relations is surprisingly low. AI does not provide any tools for the description and refinement of semantic links up to the granularity needed for the task or problem at hand. Moreover, although the underspecification of semantic links has been pointed out for main OBLR [4, 11], few initiatives $[2,21]$ exist that aim at providing not only a richer semantics to these links but also to propose specific operations that can be performed on them.

From the operations that can be performed on semantic relations, we are interested in control and verification, as we believe it would allow the kind verification (at least for semantic relations in ontologies in general) that [23] argues that KBS need. Furthermore, their enforcement through the properties of relations would allow to properly structure an ontology. We claim that this structuring is dependent not only on the domain but also on the task for which the resource is being built; as it is the context of the task (a specific application) the one that allows fixing the pertinent meaning features of concepts and relations.

Finally, we propose the inclusion of control and verification of semantic relations as part of a methodological framework for the development of OBLR with relational databases. By doing this we go, qualitatively, further than any other efforts [24] that have used relational databases to build a LR with a Mikrokosmos-like philosophy and structure [25] for specific applications. We are currently in the stage of: a) building a text corpus for the domain of Pneumonia; and b) developing a tool based on the one described in [24] in order to include the ideas mentioned here. Our final goal is to develop an OBLR that will be used as part of a summarization and categorization system.

\section{References}

1. Buenaga, M. et al. The SINAMED and ISIS Projects: Applying Text Mining Techniques to Improve Access to a Medical Digital Library. LNCS 4172, pp. 548-551, 2006.

2. Vaquero, A. et al. Conceptual Design for Domain and Task Specific Ontology-Based Linguistic Resources. LNCS 4275, pp. 658-668. 2006.

3. Vaquero, A. et al. Thinking Precedes Action: Using Software Engineering for the Development of a Terminology Database to Improve Access to Biomedical Documentation. LNCS 4345, pp. 207-218. 2006. 
4. Vaquero, A. Álvarez, F. and Sáenz, F. A Review of Common Problems in Linguistic Resources and a New Way to Represent Ontological Relationships. Electronic Journal of the Argentine Society for Informatics and Operations Research, Vol. 7(1), ISSN 1514 - 6774, pp. 1-11. 2007.

5. Yoo, J., Catanio, J. and Ravi, P. Relationship Analysis in Requirements Engineering. Requirements Engineering, Vol. 9(4), pp. 238-247. 2004.

6. Chen, P. The Entity-Relationship Model - Toward a Unified View of Data. ACM Transactions on Database Systems 1(1). 1976.

7. Booch, G. Object-Oriented Development. IEEE Transactions on Software Engineering 12(12). 1986.

8. Rumbaugh, J. 1991. Object-Oriented Modeling and Design. Prentice-Hall, New Jersey.

9. Steimann, F., Gößner, J. and Mück, T. On the Key Role of Composition in Object-Oriented Model-ing. LNCS 2863, pp. 106-120, 2003.

10. Booch, G., Rumbaugh, J \& Jacobson, I. The Unified Modeling Language User Guide. Addison Wesley. 1999.

11. Kashyap, V. and Borgida, A. Representing the UMLS® Semantic Network using OWL. LNCS 2870, pp. 1-16. 2003.

12. Schulz et al. From Knowledge Import to Knowl-edge Finishing: Automatic Acquisition and Semiau-tomatic Refinement of Medical Knowledge. In Proc. of the 12th Banff Knowledge Acquisition for Knowl-edge-Based Systems Workshop - KAW'99. 1999.

13. Nirenburg, S. et al. Ontological Semantic Text Processing in the Biomedical Domain. Working Pa-per \#03-05, Institute for Language and Information Technologies, University of Maryland. 2005.

14. Hirst G. Ontology and the Lexicon. In Staab \& Studer (eds), Handbook on Ontologies, Springer-Verlag, pp. 209-230. 2004.

15. Welty, C. \& Guarino, N. Supporting Ontological Analysis of Taxonomic Relationships. Data and Knowledge Engineering, Vol. 39(1) Elsevier. 2001.

16. Bachimont, B., Isaac, A., Troncy, R. Semantic Commitment for Designing Ontologies: A Proposal. LNCS 2473, pp. 114-119. 2002.

17. Barrett, H.C. On the Functional Origins of Essentialism. Mind and Society 3(2), pp. 1-30. 2001.

18. Medin, D. \& Ortony, A. Psychological Essentialism. In S. Vosniadou \& A. Ortony (eds.) Similarity and Analogical Reasoning. Cambridge University Press, pp. 179-195. 1989.

19. Machery, E. et al. Semantics Cross-Cultural Style. Cognition 92, pp.B1-B12. 2004.

20. Rastier, F., Cavazza, M., Abeillé, A. Sémantique pour l'analyse. De la linguistique à l'informatique, Masson. 1994.

21. Russomanno, D. A Plausible Inference Prototype for the Semantic Web. Journal of Intelligent Informa-tion Systems, Vol. 26(3), pp.227-246. 2006.

22. Hahn, U., Schulz, S., Romacker, M. 1999. Part-whole Reasoning: A Case Study in Medical Ontology Engi-neering. Intelligent Systems and their applications, Vol. 14(5), pp.59-67, IEEE Computer Society.

23. Hicks, R.C. 2003. Knowledge-Based Management Sys-tem-Tools for Creating Verified Intelligent Systems. Knowledge Based Systems 16, pp. 165-171.

24. Moreno, A. and Pérez, C. 2000. Reusing the Mikrokos-mos Ontology for Concept-Based Multilingual Ter-minology Databases. In Proc. of the 2nd LREC, pp. 1061-1067.

25.Viegas, E. et al. 1999. Semantics in Action. In P. Saint-Dizier (ed.), Predicative Forms in Natural Language and in Lexical Knowledge Bases, Kluwer, pp. 1-33. 